\title{
High Resolution Sapphire Bragg Backscattering Monochromator
}

\author{
P. van der Linden ${ }^{1}$, H.-C. Wille ${ }^{1}$, Yu. V. Shvyd'ko \\ ${ }^{I}$ European Synchrotron Radiation Facility, B.P. 220, F-38043Grenoble, France \\ ${ }^{2}$ Argonne National Laboratory, Advanced Photon Source, Argonne, Il-60439, USA
}

\begin{abstract}
We present a temperature stabilised high resolution sapphire backscattering monochromator. The device consists of a sapphire crystal inside a cold nitrogen gas cooled, temperature stabilised chamber with a passively temperature stabilised screen. The achieved temperature stability of $\pm 2 \mathrm{mK}$ allows for an energy resolution of $\Delta \mathrm{E} / \mathrm{E} \leq 10^{-7}$ at energies in the range of 30-70 keV. The device was developed for nuclear resonant scattering above $30 \mathrm{keV}$, where appropriate solutions did not exist until now.
\end{abstract}

Keywords: X-ray monochromator; $\mathrm{x}$-ray spectroscopy; Mossbauer effect; $\mathrm{x}$-ray nuclear scattering PACS: $29.20 . \mathrm{Lq}, 29.30 . \mathrm{Kv}, 41.50 .+\mathrm{h}, 41.85 . \mathrm{Si}, 76.80 .+\mathrm{y}$

\section{MONOCHROMATOR}

The device has been developped for nuclear resonant scattering of synchrotron radiation, where (sub-)meV monochromatization around the fixed nuclear resonant energies of the relevant isotopes is required. To achieve an angular acceptance of Bragg reflections that covers the beam divergence at third generation synchrotron sources (about $10 \mu \mathrm{rad}$ at $\mathrm{E}=37 \mathrm{keV}$ ) the backscattering geometry was chosen. In this case the number of adequate reflections in silicon is too small to find reflections for the fixed nuclear transition energies. Furthermore the reflectivity of silicon strongly decreases for $\mathrm{E}>30 \mathrm{keV}$. Therefore sapphire $\left(\alpha-\mathrm{Al}_{2} \mathrm{O}_{3}\right)$ crystals are used. Details and all relevant informations concerning the X-ray operating principles can be found in [1]. The monochromator has been developed on the basis of a protoype which has been built at the University of Hamburg. Although the x-ray operating principle remains the same, the cryogenic operation of the monochromator has been changed. The basic specifications have remained the same: temperature range $90 \mathrm{~K}$ to room temperature, temperature stability $2 \mathrm{mK}$, optical access in/out in a 20 degree cone. The liquid nitrogen cryogen used to cool the cryostat has been changed for cold nitrogen gas [3] to reduce the vibration level to a minimum. This has also reduced the liquid nitrogen consumption by a factor of ten from 100 liter per day to 10 liter per day.

The cold nitrogen gas enters the cryostat and first passes through an outer cooling shield to absorb the heat load coming from radiation and the support struts. This provides a stable low temperature environment for the inner chamber containing the sapphire crystal. The gas then passes through the wall of the inner chamber where it is brought to the desired temperature before it enters the inner chamber crystal space. Both outer and inner shield as well as the crystal holder are equipped with Pt100 thermometers; both shields are equipped with a wire wound manganine heater. The active temperature control by computer was also developed at the University of Hamburg. 


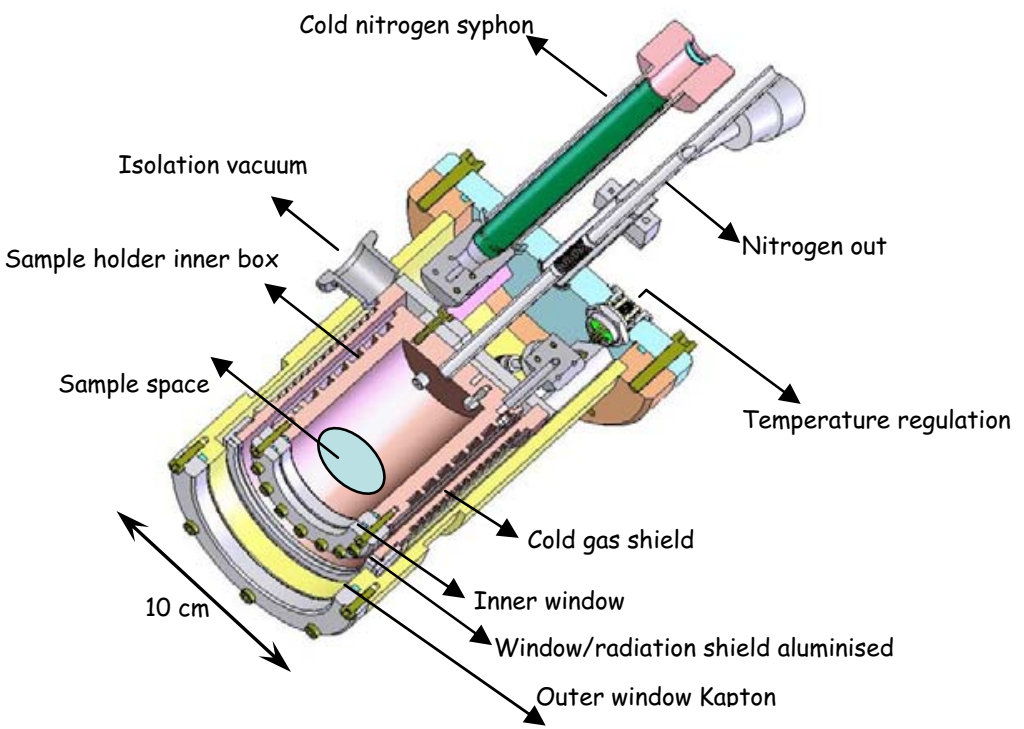

FIGURE 1. A cut view of the monochromator.

\section{RESULTS AND DISCUSSION}

A first nuclear forward scattering experiment on ${ }^{121} \mathrm{Sb}$ at $37.13 \mathrm{keV}$ has been performed [3] using the prototype and first nuclear inelastic scattering on Sb compounds was performed using the actual monochromator[4]. As the Bragg angle is fixed in backscattering geometry, energy changes are performed by changing the crystal lattice plane distance making use of the thermal expansion of the crystal. To perform linear energy scans in the meV regime the crystal temperature is linearely sweeped. For the used (15 13 -28 14) reflection in sapphire a change in $\mathrm{T}$ of $1 \mathrm{~K}$ corresponds to a change in $\mathrm{E}$ of $-60 \mathrm{meV}$. The crystal temperature and the difference of the set and the crystal temperature during a T-sweep are shown in Fig. 2.

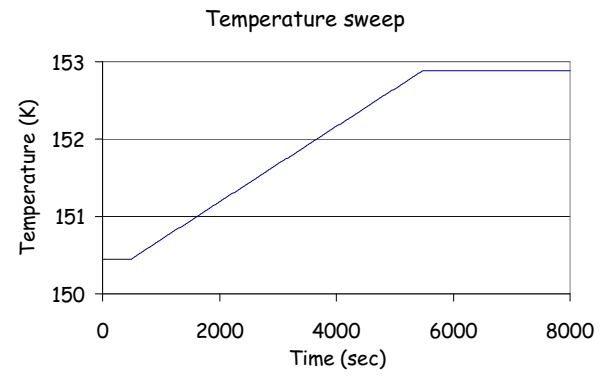

Difference Tset- Tmeasure

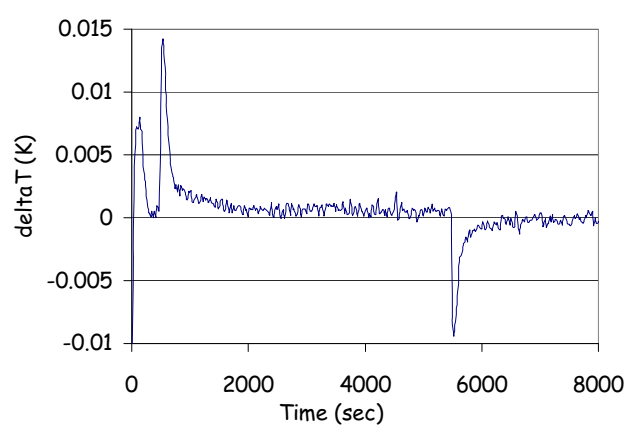

FIGURE 2. Temperature sweep and stability during the sweep. 
At the start and the end of the sweep peaks of $10 \mathrm{mK}$ arise, during the sweep and while the temperature remains stable the deviations from the setpoint are smaller than $2 \mathrm{mK}$. Figure 3 shows the measured energy resolution of the monochromator at $37.13 \mathrm{keV}$. Though the width of $8 \mathrm{meV}$ is much larger than the theoretical value $(0.4 \mathrm{meV})$ the achieved energy resolution of $2 * 10^{-7}$ allows to perform nuclear resonant scattering experiments and is unique in the energy regime above $30 \mathrm{keV}$ to our knowledge.

We are currently investigating if the peak broadening is due to dislocations in the crystal or thermal gradients within the crystal, for example thermal bump as seen in silicon monochromators.

The high resolution sapphire bragg backscattering monochromator has proven that it is easy to handle, and it yields stable temperatures even during the sweep. It is economical with cryogen, during a one week experiment the nitrogen vessel does not need to be refilled so the experiment can run continuously. Within one working day it is possible to change the crystal, cool down and adjust the monochromator to a new reflection. This is remarkably fast for such an accurate device. Once the initial test phase is concluded it will become standard equipment on the ESRF ID18 Nuclear Resonant Scattering Beamline.

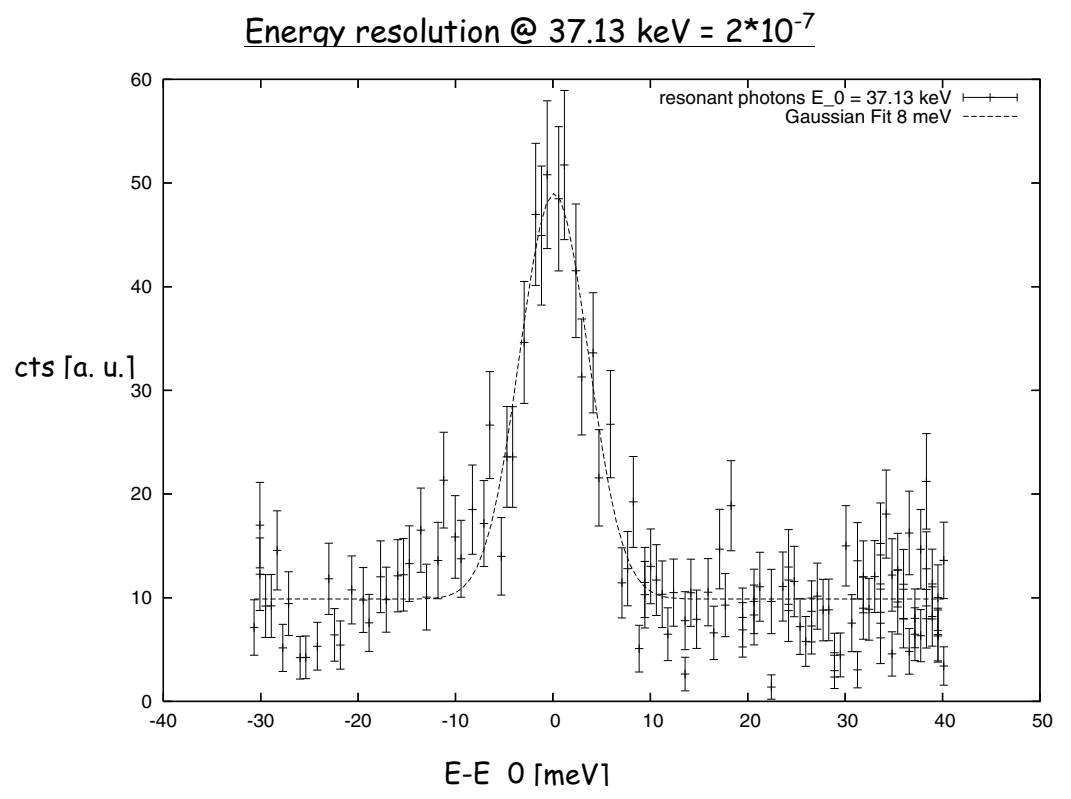

FIGURE 3. Measured energy resolution of the (15 13 -28 14) reflection in sapphire, 8 meVat $37.13 \mathrm{keV}$.

\section{ACKNOWLEDGMENTS}

ESRF, Grenoble, France: Rudolf Rüffer, Alexander Chumakov, Olaf Leupold, Andrea Monaco, Ilia Sergeev, Jean-Philippe Celse, Svetoslav Stankov, Thomas Roth, Ulrich Ponkratz, Cornelius Strohm.

University of Hamburg, Germany: Erich Gerdau, Martin Lucht, Hans Dierk Rüter

APS Argonne, USA: Esen Ercan Alp

\section{REFERENCES}

1. Yu.V. Shvyd'ko, X-Ray Optics, Springer Ser. Optical Science, Vol 98, Springer Verlag Berlin 2004

2. R. Steinmann, P. van der Linden, proceedings MEDSI 2006, to be published

3. H.-C. Wille, Yu.V. Shvyd'ko, E.E. Alp, H.D. Rüter, O. Leupold, I. Sergueev, R. Rüffer, A. Barla and J.P. Sanchez, Europhys. Letters, 74 No. 1, 170 (2006)

4. H.-C. Wille, Yu.V. Shvyd'ko et al., to be published 